\title{
Advances in ultrasonography of urinary tract obstruction
}

\author{
J. F. Platt \\ Department of Radiology, University of Michigan Hospital, 1500 E. Medical Center Drive, B1D520, Ann Arbor, MI 48109-0030, USA
}

Ultrasound has many ideal attributes as a method for detecting urinary obstruction. It is noninvasive, quick, portable, requires neither radiographic contrast media nor ionizing radiation and is relatively inexpensive. Conventional ultrasound provides anatomic information regarding obstruction, primarily a dilated collecting system. In some cases, ultrasound may reveal the level and cause of obstruction. Traditional ultrasound does not, however, provide physiologic or functional data regarding obstruction. Numerous investigations have attempted to address these limitations with the addition of a duplex Doppler ultrasound study.

\section{Physiology of renal obstruction}

The physiology of obstruction is complicated because acute complete unilateral obstruction may differ from acute complete bilateral obstruction, and both kinds differ from chronic obstruction and different degrees of partial obstruction. Because the largest volume of work has been devoted to acute unilateral obstruction, this is a logical starting place to consider the physiology of urinary tract obstruction.

There is a well-documented elevation of collecting system pressure with the onset of acute complete obstruction [1, 2]. Very elevated intrapelvic pressures $(>50 \mathrm{mmHg}$ ) can be reached quickly, with the rate of increase depending on the rate of urine flow at the onset of the obstruction. A recent review of renal obstruction by Cronan has isolated increased pressure as the main culprit in renal obstruction [3]. However, with complete renal obstruction, there is a return of renal pelvic pressure to normal levels, sometimes soon after the onset of obstruction [4-7]. This fact has led investigators to search for other critical factors such as altered renal blood flow.

Hemodynamic changes with acute complete obstruction have been well documented. At least three distinct phases of renal blood flow alteration shortly after the onset of obstruction have been described. In the first phase, there is a very transient increase in renal blood flow likely due to afferent arteriolar dilatation, perhaps mediated by prostaglandins [4, 5, 8]. In the second phase, there is an elevation of intrarenal arterial resistance likely due to circulating vasoconstrictor factors, leading to reduced renal blood flow. This phase generally begins $3-5 \mathrm{~h}$ after the onset of obstruction $[4,5]$. The third phase of obstruction, with onset at 18-24 h, is profound intrarenal vasoconstriction, resulting in reduced renal blood flow, which occurs when intratubular pressures are decreasing or even normalized [4, 5, 8, 9]. The decrease in pressure is more marked in unilateral obstruction than in bilateral obstruction. Hence, from this stage on, considerable renal damage occurs in the face of normalizing pressures, leading some to theorize that the majority of renal damage is due to elevated renal vascular resistance and vasoconstriction. This theory is supported by functional data showing a reduced GFR immediately after the onset of obstruction, which continues to decrease in the face of normalizing tubular pressures and intrarenal vasoconstriction $[4,5]$.

At what point acute obstruction becomes chronic obstruction is neither well defined nor universally agreed on. With more chronic obstruction, persistent elevation of vascular resistance and decreased renal blood flow eventually result in pathologic changes. These structural changes include tubular atrophy, interstitial widening, fibrosis, and nephron loss. There is marked dilatation of the collecting system and renal parenchymal atrophy.

Recovery of renal function after relief of obstruction is another topic about which important physiologic data is available. Rapid relief of acute obstruction appears to be a completely reversible condition with no significant long-term complications [1]. However, animal data suggest that untreated obstruction for 4-7 days results in a variable but real loss of renal function [10, 11]. Even after release of obstruction, renal function may remain decreased for days [1]. Renal vasoconstriction and elevated renal vascular resistance also persists for days af- 
ter relief of obstruction, perhaps contributing to the slow recovery of function.

The preceeding overview has generally focused on complete obstruction. Partial obstruction, although more common clinically, has received much less attention in both animal and human investigations. Clinically significant partial obstruction is almost always a chronic disease, and if the obstruction is of a certain degree, changes similar to complete obstruction can occur. Prediction of which partial obstruction instances are significant and can lead to renal atrophy, and functional loss is at the center of much of the imaging performed in patients with known partial obstruction.

\section{Doppler ultrasound}

Doppler assessment of intrarenal resistance may be an accurate marker for significant obstruction because many investigators believe that hemodynamic changes are central to renal damage from obstruction.

Doppler study of small intrarenal vessels, although not technically difficult, requires proper technique to obtain useful measurements. Most studies have concentrated on the study of distal intrarenal vessels, which are not actually seen during the examination but are detected by sampling the Doppler spectrum at the corticomedullary junction or along borders of medullary pyramids. In general, these small vessels have low flow velocities and, therefore, relatively small frequency shifts. To detect these low velocities, the wall filter should be set as low as possible. Of even greater importance is using the smallest possible frequency range (minimum pulse repetition frequency) that does not produce aliasing [12]. These simple technical adjustments are crucial, and failure to make them often results in Doppler waveforms that barely deviate from the baseline and in which measurements will be grossly inaccurate [12].

To characterize the intrarenal Doppler waveform, most investigators have used the resistive index (RI). This easily calculated parameter is defined as ([peak systolic shift - minimum diastolic shift]/peak systolic shift). Increases in downstream resistance result in a relative reduction in diastolic flow compared with systolic flow and thus in an elevated RI. Hence, the RI can be used to estimate the state of renal arterial resistance.

Several recent studies have investigated the normal intrarenal RI values; the majority suggest 0.70 as a reasonable upper limit for the normal RI [12-14]. Conditions other than renal disease also can affect the RI. Significant hypotension, a markedly decreased heart rate, and a perinephric or subcapsular fluid collection can elevate the RI $[15,16]$. In addition, children, especially the neonate and infant, can normally have renal RIs that would be elevated by adult standards [17]. Ag- ing can also alter the RI in "normal" patients; however, this elevation may, in fact, accurately indicate the true loss of function noted in the senescent kidney, which is often not reflected in an elevated creatinine level $[12,18]$.

Numerous clinical and laboratory studies over the past few years have evaluated the role of duplex Doppler in the imaging of obstruction [12-14, 19-32]. These studies have clearly demonstrated that obstruction can produce an elevated RI (Fig. 1). Because intrarenal arterial resistance increases with obstruction, the RI should reflect this altered resistance. To identify an elevated RI, different criteria have been suggested, including elevation above a threshold value $(0.70)$, an interrenal RI difference greater than 0.06-0.10 with unilateral dilatation, and an abnormal RI response to a diuretic challenge [25-27]. The latter two criteria appear most helpful in the pediatric age group, in equivocal partial obstruction, and in patients with underlying renal medical disease. By whatever criteria, Doppler abnormalities can be observed with acute, chronic, complete, partial, adult, and pediatric obstruction.

A second crucial observation is that the RI is a separate and distinct parameter from collecting system dilatation. Obstruction without pyelocaliectasis (such as very acute obstruction) can elevate the RI, and severe dilatation without obstruction will often not elevate the RI. Because the RI and assessment of collecting system dilatation are distinct and separate parameters, they will have different false positive and negative examinations. Therefore, renal Doppler analysis is most useful when analyzed in conjunction with traditional ultrasound assessment of the collecting system.

The controversial issues with renal Doppler are similar to those of many tests assessing the significance of possible renal obstruction. Experience suggests that Doppler analysis can be quite helpful in distinguishing obstructive from nonobstructive pyelocaliectasis, with reported accuracies of 77-96\% [12-14, 19-32]. However, a few recent studies have reported much lower accuracies [33, 34]. These studies have focused on the clinical setting of acute renal colic, which will be discussed later [33, 34]. Less objective data is present on RI accuracy in assessment of partial obstruction, which can be a difficult topic to analyze due to lack of a wellestablished "gold standard.",

Partial obstruction highlights the fact that Doppler analysis attempts to provide physiologic rather than anatomic information. Many cases are observed with areas of mild ureteric narrowing or kinking and accompanying normal findings on Doppler examinations. The normal RI likely reflects the lack of obstruction of sufficient degree to produce vasoconstriction, elevate renal resistance, and cause renal atrophy. Although a normal RI argues against significant physiologic obstruction, it does not imply that a ureter is free of mild narrowing; 

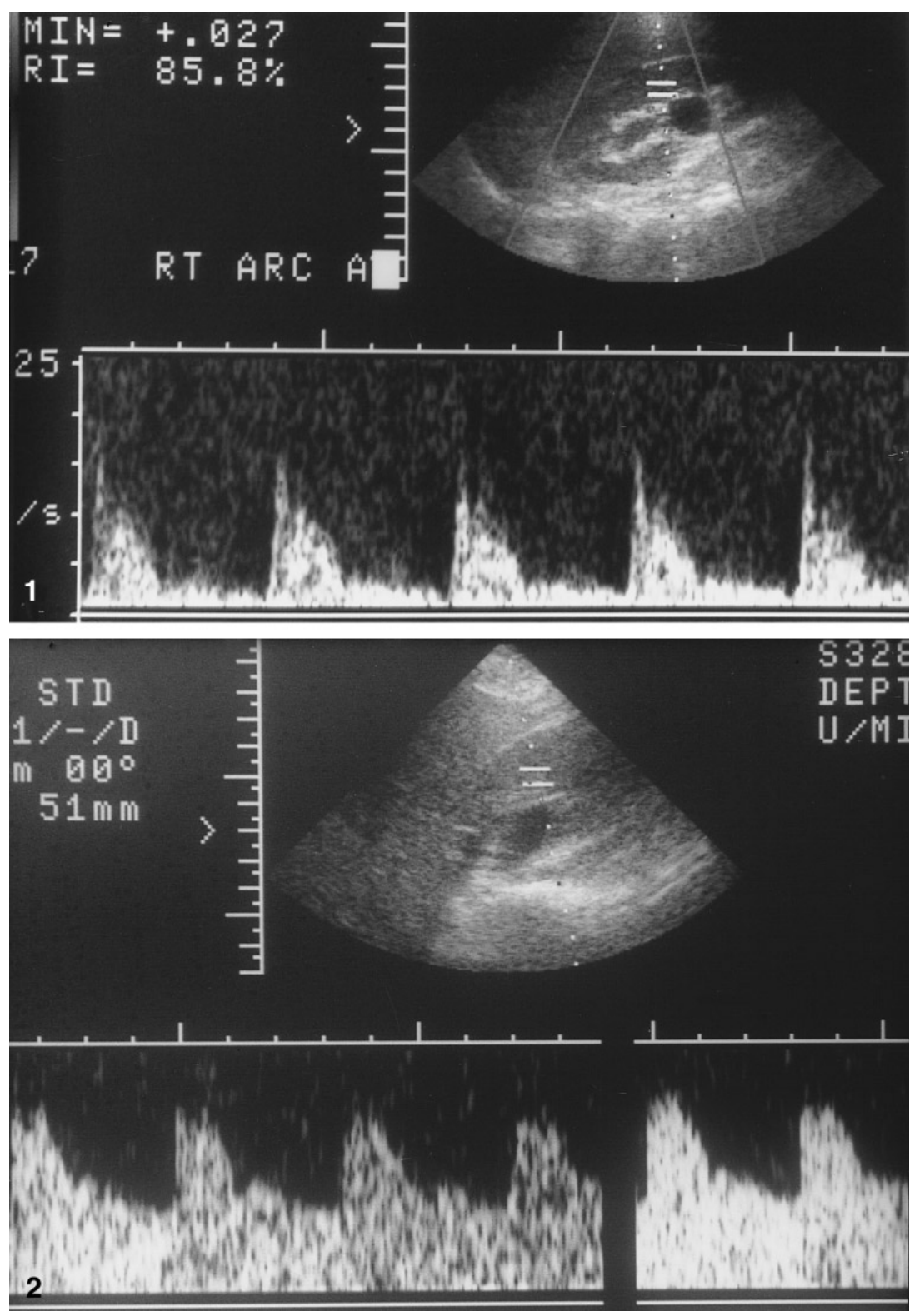

Fig. 1. Doppler of right renal arterial system. Right renal obstruction due to distal ureteral calculus produces RI elevation.

Fig. 2. Doppler tracing from right kidney in pregnant woman (at 24 weeks) with clinical renal colic. Collecting system dilatation is noted, but the RI is normal. Subsequent limited intravenous pyelogram confirmed no mechanical obstruction. the renal Doppler examination alone does not suffice if precise anatomic information is required.

As the frequency of finding a dilated collecting system increases, the value of renal Doppler analysis also increases. For example, asymptomatic pregnant women often have dilatation of their renal collecting systems or "hydronephrosis of pregnancy." Three recent studies agree that this hydronephrosis of pregnancy does not typically produce RI elevation (Fig. 2) [35-37]. Hence, an abnormal renal Doppler suggests true mechanical obstruction, such as a ureteral calculus.

Another group of patients with frequent collecting system dilatation are those with prior obstruction relieved by an internal ureteral stent. With relief of ob- struction, the RI typically returns to baseline levels in a matter of days. The RI decrease occurs even if the pyelocaliectasis persists, which is often the case in obstructed patients who undergo stent placement. A recent study has found Doppler analysis to be valuable in determining stent obstruction or patency (Fig. 3) [38]. For the same reasons, renal Doppler follow up is often helpful after surgical intervention for obstruction. Normalization of the RI implies resolution of the obstruction, even if collecting system dilatation persists.

Use of Doppler criteria in the pediatric age group (especially those younger than 4 years) can be more difficult [17]. Elevated RIs are commonly observed in normal kidneys in infants and neonates [17]. Despite 

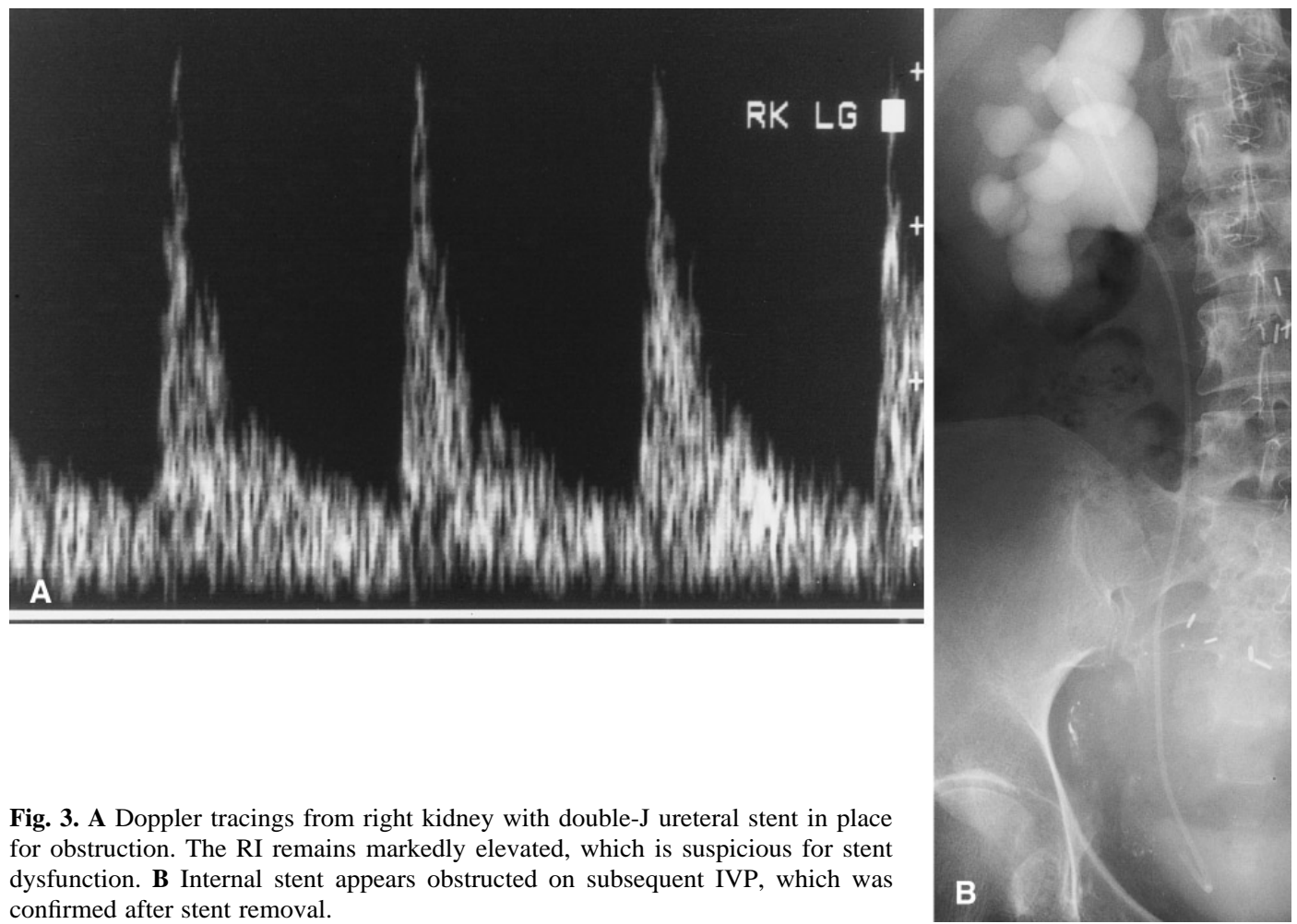

Fig. 3. A Doppler tracings from right kidney with double-J ureteral stent in place for obstruction. The RI remains markedly elevated, which is suspicious for stent dysfunction. B Internal stent appears obstructed on subsequent IVP, which was confirmed after stent removal.

this limitation, excellent results have been reported. However, relying on a simple threshold criterion for an abnormal RI is less desirable in the pediatric age group [22, 24]. Instead, kidney-to-kidney comparisons/ratios and comparison to baseline studies are more helpful. In addition, pharmacologically stimulated Doppler studies may be useful in the pediatric kidney [26, 27].

There is a growing body of literature supporting the benefits of adding a diuretic challenge to the standard renal Doppler examination [25-27]. These studies suggest that there is an increase in the renal RI after diuretic administration when significant obstruction is present. No change in the RI is noted in normal kidneys, in those with nonobstructive dilatation, and in those with milder partial obstruction. Several papers have reported on the utility of the diuretic Doppler examination in the pediatric age group, including patients with UPJ obstructions [26, 27]. In the adult population, Mallek et al. found renal RI elevation in obstructed kidneys but not in patients with normal kidneys or those with nonobstructive dilatation [25]. Their data analysis revealed an RI of 0.69 to be an optimal threshold level, which is almost identical to the 0.70 level derived from our earlier work $[13,25]$. They found an even higher accuracy $(95 \%)$ when diuretic Doppler was added to the examination. The renal RI increased in response to diuretic only when significant obstruction was present. A threshold value of RI $>0.75$ was suggested for a postdiuretic renal RI to indicate significant obstruction. Further work on pharmacologically stimulated Doppler will likely be forthcoming in the near future, thereby helping to define the proper role for renal Doppler in the imaging of urinary tract obstruction. It is unfortunate that the debate over renal Doppler utility in the radiologic literature has largely fallen to the setting of acute renal colic. Here, the issues are largely those of anatomic obstruction and are not of functionally or physiologically significant obstruction.

\section{Acute renal colic}

The proper imaging protocol for patients with possible renal colic is controversial. The issues in this setting are largely those of anatomic obstruction. The question to be answered is, Does the patient have a renal or ureteral etiology for pain? Many investigators still consider the IVP as the "gold standard" for acute renal colic.

Numerous clinical and laboratory studies over the past few years have evaluated the role of duplex Doppler in the evaluation of acute obstruction $[14,19,23$, 29-34]. Some studies have reported on a few cases of acute obstruction as part of larger studies dealing with all types of possible obstruction. More recently, several studies have focused more directly on acute renal colic and obstruction with duplex Doppler ultrasound (Fig. 4). 

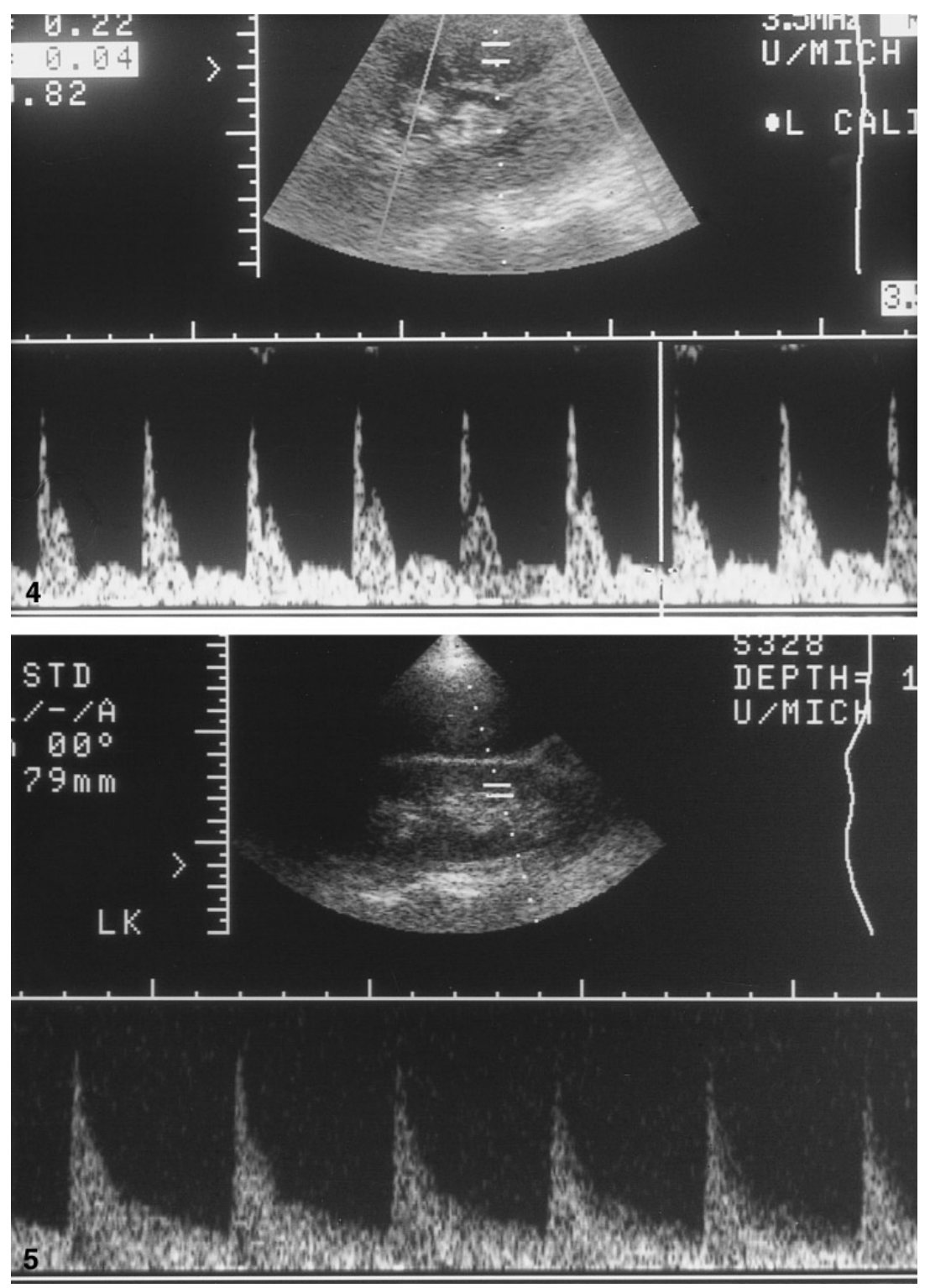

Fig. 4. Doppler tracing of kidney in patient with acute renal colic. Conventional sonography reveals only mild collecting system dilatation; however, the RI is already markedly elevated after only $8 \mathrm{~h}$ of symptoms.

Fig. 5. Doppler tracing of left kidney in patient with $6 \mathrm{~h}$ of left flank pain. Conventional sonographic study was normal; however, the $\mathrm{RI}$ is elevated due to acute obstruction.

In a study of 12 patients with acute obstruction, Sauvain et al. found RI elevation $(\geq 0.70)$ in $100 \%$ of patients [14]. They found an RI difference between obstructed kidney and nonobstructed kidney $(\Delta \mathrm{RI}) \geq 0.10$ in all 12 patients.

Rodgers et al. reported a mean RI of $0.70 \pm 0.06$ in 14 acutely obstructed kidneys, which was significantly higher than the RI in the contralateral normal kidney and normal kidneys [30]. The $\Delta \mathrm{RI}$ was significantly higher in patients with acute obstruction $(0.08 \pm 0.04)$ than in normal patients $(0.03 \pm 0.02)$. Rodgers et al. described five kidneys with a normal conventional ultrasound despite IVU-proven obstruction [30]. Four of these had an abnormal Doppler study.

Brkljacic et al. studied 21 patients with acute obstruction and 33 normal patients [19]. Obstructed kid- neys overall had a mean RI $(0.71 \pm 0.04)$ greater than the contralateral normal kidney $(0.59 \pm 0.03)$ and normal kidneys $(0.59 \pm 0.04)$. Brkljacic et al. found the $\Delta \mathrm{RI}$ to be a better criterion for obstruction than absolute RI elevation [19]. The $\Delta \mathrm{RI}$ was $0.12 \pm 0.03$ in obstructed kidneys and $0.01 \pm 0.01$ normal kidneys. Using a $\Delta$ RI threshold of 0.08 , Brkljacic et al. found a $91 \%$ sensitivity and $100 \%$ specificity for acute obstruction diagnosis [19]. They also found six patients with acute obstruction who had a $\Delta \mathrm{RI} \geq 0.08$ but a normal conventional ultrasound and four patients with a normal ultrasound but obstruction indicated by an RI of $\geq 0.70$ [19].

At our institution, we reported on 23 patients with acute unilateral obstruction [29]. The mean RI $(0.77 \pm$ 0.07 ) was significantly higher in the obstructed kidney 
than in the contralateral normal kidney $(0.60 \pm 0.04)$. The Doppler study was $87 \%$ sensitive for acute obstruction [29]. We encountered four instances of acute renal obstruction with a normal conventional ultrasound and an abnormal Doppler study [29].

In addition to the five clinical studies discussed earlier, a recent animal study has found encouraging Doppler results for duplex Doppler evaluation of acute obstruction [32]. In a dog model, Ulrich et al. found RI elevations as early as $1.5 \mathrm{~h}$ and at reliable levels by $4 \mathrm{~h}$ [32]. They also found a comparison of RI values between obstructed and normal kidneys within a given dog to be most helpful [32]. They reported an RI ratio of $\geq 1.15$ to be $100 \%$ sensitive and specific for acute obstruction of $4 \mathrm{~h}$ duration [32].

Altogether, these clinical and laboratory studies show that acute obstruction produces RI elevation as compared with the contralateral normal kidney and normal kidneys. The difference in RI between the obstructed kidney and other normal kidneys is often more helpful than just an elevation above a specific threshold. Lastly, Doppler can detect changes of acute obstruction at a time when conventional ultrasound is normal (Fig. 5).

However, two recent studies have reported disappointing results for duplex Doppler of acute obstruction, thus sparking controversy in the recent radiologic literature $[33,34]$. Deyoe et al. found an abnormal Doppler study in only $30 \%$ (3/10) of high-grade acute obstructions and none of 14 cases of acute partial obstruction [33]. Tublin et al. reported on 32 patients with acute colic, 19 of whom proved to have acute obstruction [34]. Although they found significant RI elevation and elevation of the $\Delta \mathrm{RI}$ with acute obstruction, they found no useful threshold levels for these Doppler parameters.

Renal duplex Doppler sonography detects the hemodynamic changes that accompany significant obstruction, and, therefore, it is primarily a functional test. Therefore, the same clinical situations in which one might consider using a Whitaker test or a diuretic renogram are those most ideal for renal Doppler imaging, whereas clinical questions usually answered by a retrograde pyelogram or an IVU are less optimal for renal Doppler imaging. The clinical setting of acute renal colic largely poses an anatomic obstruction question: Does a patient with acute pain have any renal or ureteral etiology for this pain? The most widely accepted imaging test used in this setting, the IVU, deals primarily with anatomic obstruction. The clinical question raised by renal colic would not be adequately addressed by the diuretic renogram or Whitaker test.

What then should be the role of renal Doppler imaging in the setting of suspected acute renal colic? There are at least three situations in which renal Doppler imaging can contribute significantly to diagnosis and man- agement. First, for situations in which intravenous contrast administration is undesirable (such as pregnancy, contrast agent allergy, and renal dysfunction), sonography is often used. The use of renal Doppler study can be helpful, regardless of what the standard sonographic study shows. If pyelocaliectasis of a symptomatic kidney is revealed, an abnormal RI confirms that obstruction is present and is likely of functional significance. However, normal renal Doppler findings accompanying pyelocaliectasis suggests that an anatomic obstruction is probably present but that the obstruction is not as significant functionally; hence, a more conservative management approach may be indicated. If a normal standard sonographic examination is obtained, a Doppler study is still indicated because RI changes can be observed before pyelocaliectasis, thereby improving the sensitivity of conventional sonography for acute obstruction.

The second situation occurs at institutions at which sonography is used as a primary technique for evaluating acute renal colic. In such situations, the addition of a Doppler study is clearly indicated. As discussed above, the literature suggests that up to $20 \%$ of cases of acute obstruction have Doppler abnormalities, despite normal gray-scale sonographic findings. Therefore, when sonography serves as a replacement for IVU, a renal Doppler study is necessary to improve the sensitivity for early obstruction and to provide functional information regarding an obstructed kidney.

Third, at medical centers at which IVU remains the primary imaging technique for acute renal colic and when contrast agent administration is not contraindicated, a renal Doppler study should be used selectively, usually with regard to decisions concerning patient management.

If the suggested recommendations are followed, a proper role for renal Doppler imaging imaging in the setting of acute renal colic will continue to evolve.

\section{References}

1. Gillenwater JY. The pathophysiology of urinary obstruction. In: Walsh PC, Gittes RF, Perlmutter AD, et al., eds. Campbell's Urology, 5th ed. Philadelphia: WB Saunders, 1986:542-544

2. Moody TE, Vaughan ED Jr, Gillenwater JY. Relationship between renal blood flow and ureteral pressure during 18 hours of total unilateral ureteral occlusion. Implications for changing sites of increased renal resistance. Invest Urol 1975;13:246-251

3. Cronan JJ. Contemporary concepts in imaging urinary tract obstruction. Radiol Clin North Am 1991;29:527-542

4. Klahr S. New insights into the consequences and mechanisms of renal impairment in obstructive nephropathy. Am J Kidney Dis 1991;18:689-699

5. Klahr S. Pathophysiology of obstructive nephropathy: a 1991 update. Sem Nephrol 1991;11:156-168

6. Leahy AL, Ryan PC, Mcentee GM, et al. Renal injury and recovery in partial ureteric obstruction. J Urol 1989;142:199-203 
7. Michaelson G. Percutanous puncture of the renal pelvis, intrapelvic pressure and the concentrating capacity of the kidney in hydronephrosis. Acta Med Scand Suppl 1974;559:1-26

8. Yarger WE, Schocken DD, Harris RH. Obstructive nephropathy in the rat: possible roles for the renin-angiotensin system, prostaglandins, and thromboxanes in postobstructive renal function. $J$ Clin Invest 1980;65:400-412

9. Holden D, George NJR, Rickards D, et al. Renal pelvis pressures in human chronic obstructive uropathy. Br J Urol 1984;56:565570

10. Fallon B. Functional recovery in a kidney after prolonged complete ureteric obstruction. Br J Urol 1977;49:72

11. Kerr WS. Effect of complete ureteral obstruction for one week on kidney function. J Appl Physiol 1954;6:762-772

12. Platt JF. Duplex doppler evaluation of native kidney dysfunction: obstructive and nonobstructive disease. AJR 1992;158:10351042

13. Platt JF, Rubin JM, Ellis JH. Distinction between obstructive and nonobstructive pyelocaliectasis with duplex Doppler sonography. AJR 1989;153:997-1000

14. Sauvain JL, Bourscheid D, Pierrat V, et al. Duplex Doppler sonography of intrarenal arteries. Normal and pathological aspects. Ann Radiol 1991;34:237-247

15. Mostbeck GH, Gossinger HD, Mallek R, et al. Effect of heart rate on Doppler measurements of resistive index in renal arteries. $\mathrm{Ra}$ diology 1990;175:511-513

16. Pozniak MA, Kelcz F, Stratta RJ, et al. Extraneous factors affecting resistive index. Invest Radiol 1988;23:899-904

17. Bude RO, DiPietro MA, Platt JF, et al. Age dependency of the renal resistive index in healthy children. Radiology 1992;184: $469-473$

18. Terry JD, Rysavy JA, Frick MP. Intrarenal Doppler: characteristics of aging kidneys. J Ultrasound Med 1992;11:647-651

19. Brkljacic B, Drinkovic I, Sabljar-Matovinovic M, et al. Intrarenal duplex Doppler sonographic evaluation of unilateral native kidney obstruction. J Ultrasound Med 1994;13:197-204

20. Chen JH, Pu YS, Liu SP, et al. Renal hemodynamics in patients with uropathy evaluated by duplex Doppler sonography. J Urol 1993; 150:18-21

21. Fung LC, Steckler RE, Khoury AE, et al. Intrarenal resistive index correlates with renal pelvis pressure. J Urol 1994;152:607-611

22. Gilbert R, Garra B, Gibbons MD. Renal duplex Doppler ultrasound: an adjunct in the evaluation of hydronephrosis in the child. J Urol 1993;150:1192-1194

23. Gottlieb RH, Luhmann K, Oates RP. Duplex ultrasound evaluation of normal native kidneys and native kidneys with urinary tract obstruction. J Ultrasound Med 1989;8:609-611
24. Kessler RM, Quevedo H, Lankau CA, et al. Obstructive vs nonobstructive dilatation of the renal collecting system in children: distinction with duplex sonography. AJR 1993;160:353-357

25. Mallek R, Bankier AA, Etele-Hainz A, et al. Distinction between obstuctive and nonobstructive hydronephorsis: value of diuresis duplex Doppler sonography. AJR 1996;166:113-117

26. Ordorica RC, Lindfors KK, Palmer JM. Diuretic Doppler sonography following successful repair of renal obstruction in children. J Urol 1993;150:774-777

27. Palmer JM, Lindfors KK, Ordorica RC, et al. Diuretic Doppler sonography in postnatal hydronephrosis. J Urol 1991;146:605608

28. Platt JF, Rubin JM, Ellis JH, et al. Duplex Doppler ultrasound of the kidney: differentiation of obstructive from non-obstructive dilatation. Radiology 1989;171:515-517

29. Platt JF, Rubin JM, Ellis JH. Acute renal obstruction: evaluation with intrarenal duplex Doppler and conventional US. Radiology 1993; $186: 685-688$

30. Rodgers PM, Bates JA, Irving HC. Intrarenal Doppler ultrasound studies in normal and acutely obstructed kidneys. $\mathrm{Br} J$ Radiol 1993;65:207-212

31. Sauvain JL, Pierrat V, Chambers R, et al. Ultrasound and pulsed Doppler in the study of the arteries of the renal parenchyma during obstructive syndromes and dilatation of the kidney. J Radiol 1989;70:389-398

32. Ulrich JC, York JP, Koff SA. The renal vascular response to acutely elevated intrapelvic pressure: resistive index measurements in experimental urinary obstruction. J Urol 1995;154: $1202-1204$

33. Deyoe LA, Cronan JJ, Breslaw BH, et al. New techniques of ultrasound and color Doppler in the prospective evaluation of acute renal obstruction. Do they replace the intravenous urogram? Abdom Imaging 1995;20:58-63

34. Tublin ME, Dodd GD III, Verdile VP. Acute renal colic: diagnosis with duplex Doppler US. Radiology 1994;193:697-701

35. Hertzberg BS, Carroll BA, Bowie JD, et al. Doppler US assessment of maternal kidneys: analysis of intrarenal resistivity indexes in normal pregnancy and physiologic pelvicaliectasis. $R a$ diology 1993;186:689-692

36. Nazarian GK, Platt JF, Rubin JM, et al. Renal duplex Doppler sonography in asymptomatic women during pregnancy. J Ultrasound Med 1993;12:441-444

37. Schreiber RS, Kelley RB. Doppler assessment of renal resistive indices in patients with pregnancy induced hydronephrosis. JDMS 1992;8:68-71

38. Platt JF, Ellis JH, Rubin JM. Assessment of internal ureteral stent patency in patients with pyelocaliectasis. AJR 1993;161:87-90 